\title{
UM FRAMEWORK PARA A ANÁLISE DA INTERATIVIDADE DE SITES DE E-COMMERCE DO RAMO DA MODA EM SMARTPHONE
}

\author{
Camila Barth Paiva \\ Universidade do Vale do Itajaí - Univali \\ camilabarth@univali.br \\ Vinicius Gadis Ribeiro \\ Centro Universitário Ritter dos Reis - UniRitter \\ vinicius@uniritter.edu.br
}

Resumo: Este estudo propõe um quadro referencial para a análise da interatividade de sites de e-commerce do ramo da moda especificamente, vestuário - via smartphone. Para tanto, foram investigados conceitos e especificidades existentes na literatura sobre design de interação, interatividade, especificidades de e-commerce de moda e especificidades sobre dispositivos móveis. Considerando a base teórica, utilizou-se o método de análise de conteúdo e se estabeleceu um quadro referencial para análise de interatividade de sites de e-commerce do ramo da moda-vestuário, validado por quatro especialistas da área. Como resultado, apresentam-se os fatores e critérios quanto aos níveis de interatividade identificados, assim como a proposta de um modelo específico para a análise da interatividade de sites de e-commerce.

Palavras-chave: design de interação, smartphone, e-commerce, moda, vestuário

\begin{abstract}
This study proposes a reference framework for the analysis of interactive e-commerce fashion sites - specifically, clothing - smartphone. Therefore, were investigated concepts and existing specificities in the design literature about interaction, interactivity, specifics of e-commerce and fashion specificities about mobile. Considering a theoretical basis used -if the content analysis method and established a framework reference for site interactivity analysis of the fashion business e-commerce-Clothing validated by four experts in the field. As a result, feature factors and criteria the identified levels of interactivity, so as a proposal for a specific model for site interactivity analysis e-commerce.
\end{abstract}

Keywords: Interaction design, smartphone, e-commerce, fashion, clothing 


\section{INTRODUÇÃO}

Empresas do ramo do vestuário apresentam crescimento no ramo da venda online, o que facilita aos consumidores o acesso aos produtos de diferentes marcas. Além disso, o consumo de produtos através de mobile commerce - com foco em smartphones - tem crescido consideravelmente - portanto, enfatizando cada vez mais a importância de gerar boas experiências aos consumidores, em diferentes meios para o consumo online. Desta forma, é relevante analisar a interatividade de sites de $e$ commerce do ramo da moda (vestuário) oferecidos para smartphone. Frente a isso, esse trabalho investiga os fatores e critérios que devem ser considerados em nível de interatividade para a análise de sites de e-commerce do ramo da moda-vestuário via smartphone, tendo como objetivo propor um quadro referencial para análise da interatividade de sites de e-commerce do ramo da moda-vestuário via smartphone.

O uso de dispositivos móveis tem alterado a forma como as pessoas se comunicam e interagem atualmente: é uma ferramenta que tem contribuído também para o consumo online. $O$ dispositivo móvel refere-se à conexão em tempo real, sem fio, entre o dispositivo e outros ambientes de computação, como a internet ou uma intranet (RAINER JR. e CEGIELSKI, 2011). Nesse sentido, para o m-commerce operar de maneira eficaz - do ponto de vista do usuário -, a interface do dispositivo sem fio deve funcionar de forma tão natural quanto o PC no comércio. Além disso, a velocidade da rede deve ser eficiente para que os usuários não se frustrem. Outro aspecto de interesse é a segurança, que deve ser considerada principalmente em relação à privacidade da operação e a confiança de que a transação é realizada apenas entre as partes pretendidas (STAIR e REYNOLDS, 2012).

Stair e Reynolds (2012) complementam explicando que, atualmente, os dispositivos móveis possuem limitações que complicam a acessibilidade para o uso de comércio móvel. Dentre essas, ressaltam as pequenas dimensões das telas - limitando a capacidade de exibir informações -, possuem menor potência para o processamento de dados e menor largura de banda - comparada com os computadores de mesa. Um sistema bem-sucedido e estruturado de e-commerce deve apoiar-se em múltiplos estágios, para que os consumidores vivenciem o ciclo do processo de vendas. Os autores ainda afirmam que, no sistema de comércio eletrônico, é importante oferecer ao usuário a capacidade de buscar e identificar itens, de selecionar estes itens e negociar preços, de identificar as formas de pagamento e previsão de data para a entrega, enviar o pedido ao fabricante para aquisição dos itens, realizar o pagamento pelos produtos ou serviço, obter a entrega do produto e receber apoio necessário pósvenda.

Os sites de e-commerce do ramo da moda - em especial, do vestuário - devem prever diferenciais relacionados à interação, comparados a outros tipos de segmento de e-commerce. Isso se deve ao fato de que os sites de venda online para vestuário buscam prever e atender a demanda e exigência do consumidor de moda oferecendo ferramentas que facilitem o acesso de informações sobre o produto, que permitam ao consumidor a compra eficaz e próxima da realidade de uma loja física, através do acesso de informações. Como exemplo, podem ser citados a variedade de cores, tamanho, descrição, zoom, detalhes das peças, finalização do pedido e detalhes na forma de pagamento. Entretanto, se essas informações não forem bem projetadas, a interatividade pode ser limitada, frustrando o usuário e comprometendo sua interatividade com o meio. Essas afirmações tornam-se ainda mais relevantes aliadas 
ao consumo por meio de smartphones - uma vez que se trata de um dispositivo móvel, cujo meio, o e-commerce, deverá se adaptar e atender à demanda exigida pelo consumidor.

Diante do apresentado, o estudo contribui por meio da proposta de um quadro de análise de interatividade de sites de e-commerce específicos para o ramo da modavestuário em smartphone, oferecendo aos pesquisadores da área, desenvolvedores e designers uma ferramenta que possibilite, de modo mais estruturado, levantamento e identificação de requisitos para especificação de sites de e-commerce em moda enfatizando, portanto, a preocupação do design no seu campo de pesquisa para soluções interativas nos mais diversos meios.

Considerando a base teórica, utilizou-se o método de análise de conteúdo e estabeleceu-se um quadro referencial para análise de interatividade de sites de $e$ commerce do ramo da moda-vestuário. A validação do quadro referencial deu-se através do método de inspeção, realizado por quatro especialistas da área. Para isso, realizou-se a análise da interatividade de dois sites de e-commerce, denominados na pesquisa de E-commerce $A$ e E-commerce $B$, não se expondo a identidade das empresas na pesquisa.

O presente trabalho se estrutura em cinco seções: inicialmente aborda o Design de Interação e os Níveis de Interatividade; em seguida, apresenta a metodologia aplicada no estudo; a seção seguinte aborda o resultado do estudo - que se concentra no quadro referencial de análise de interatividade - e as considerações finais.

\section{DESIGN DE INTERAÇÃO E NÍVEIS DE INTERATIVIDADE}

$O$ design de interação preocupa-se com o projeto de produtos interativos, com a criação de experiências eficazes para apoiar o modo pelo qual pessoas se comunicam e com a averiguação do modo pelo qual pessoas interagem em seu cotidiano (ROGERS; SHARP; PREECE, 2013). Observa-se que o objetivo principal, nesse âmbito, envolve o atendimento às necessidades do usuário, o que deve ocorrer da melhor forma possível. Essa demanda contempla a importância da abordagem dos conceitos vinculados aos níveis de interatividade - tratando-se de critério ligado diretamente à boa experiência do usuário.

Para Rafaeli (1988), a interatividade é entendida como um atributo natural da relação face a face e das características que definem os sistemas de duas vias de cabos, sistemas de texto eletrônico, de programação, como em jogos de vídeo interativos. O autor aborda uma definição específica de interatividade, onde trata o conceito como uma característica variável de configurações de comunicação. A interatividade é um atributo de expressão sobre o ponto em que uma determinada série de trocas de comunicação são realizadas. Dessa forma, a interatividade plena é considerada ideal para que o designer oriente seus sistemas, mas nem sempre é alcançada pelos usuários. Para isso ocorrer, a interatividade deve agir como uma estrutura composta por uma grande variedade de sistemas de comunicação, que deve permitir através de canais e meios de comunicação a substituição dos reais "participantes" no processo de comunicação. Ou seja, a interatividade é aplicada a uma ampla gama de configurações de comunicação através da não-mediada, face-aface.

Alguns conceitos relacionados ao dimensionamento que definem a interatividade, de acordo com Rafaeli (1988) em ambiente virtual, são: 
Bidirecionalidade: trata da resposta rápida e imediata, que funciona em duas direções.

Controle do usuário: trata da quantidade de atividades que o usuário pode realizar em um sistema;

Feedback: trata da resposta ou retorno que se oferece ao usuário diante da do sistema;

Inteligência artificial: Gomes apud Bortolás (2012), assim como Gonçalves e Vieira (2012, p.40), explicam o significado de inteligência virtual sob o ponto de vista de Rafaeli (1988): "a inteligência artificial pode envolver pensamento e raciocínio, quando é definida como "sistemas que pensam como seres humanos" ou quando é definida como "sistemas que pensam racionalmente. "

Transparência: Bortolás, Gonçalves e Vieira (2012, p.40) explicam que, para Rafaelli (1988), "a característica de transparência, no âmbito de interfaces digitais, pode ser entendida como a facilidade de perceber as ações que podem ser realizadas pelo usuário".

E ainda a presença social - conceito no qual o autor não expôs uma definição descritiva.

Rafaeli (1988) defende que a definição mais útil para a interatividade seria baseada na capacidade de resposta, que distingue a comunicação interativa, quase interativas (reativa), e não-interativos. A definição de interatividade reconhece três níveis pertinentes: comunicação (não-interativo), comunicação reativa (ou quaseinterativo) e comunicação e totalmente interativo.

Nesse sentido, o autor expõe que essas classes de questões devem orientar uma investigação mais aprofundada referente à construção de interatividade, e enfatiza ainda que pesquisadores devem utilizar critérios de avaliação para identificar a interatividade.

Steuer (1993) aborda como os diferentes meios de comunicação existentes podem ser classificados em termos de interatividade. Para o autor, a interatividade é definida como o alcance em que os usuários podem interagir e modificar a forma e conteúdo de um ambiente mediado em tempo real. O autor, em concordância com Rafaelli (1988) explica que, diferente do conceito dado pela maioria dos pesquisadores da área da comunicação, interatividade é uma variável ligada ao estímulo e, que é determinada pela tecnologia do meio. Esse autor complementa o conceito e propõe que a interatividade é uma variável de grande preocupação para pesquisadores em interação humano-computador e aborda três fatores que contribuem para a boa interatividade, sendo:

Velocidade: refere-se à velocidade de interação, ou o tempo de resposta, sendo uma das características mais importantes de uma mídia de sistema interativo, a interação em tempo real representa claramente o maior valor possível para esta variável: as ações de um utilizador de forma instantânea alteram o ambiente mediado por ele. Considera-se, neste caso, que a rapidez de resposta seja uma das propriedades que tornam sistemas altamente vivenciados pelos usuários (STEUER, 1993).

Alcance: refere-se ao número de possibilidades para a realização de uma ação a qualquer momento no sistema. Alcance na interatividade é determinado pelo número de atributos, que podem ser manipulados e mediados e pela quantidade de variação possível dentro de cada atributo. Steuer (1993) explica que o alcance se refere à quantidade de mudanças que pode ser efetuada pelo usuário quando mediado no 
ambiente; trata-se das dimensões específicas que podem ser alteradas, que dependem das características do determinado meio - mas que, neste processo, inclui ordenação temporal, organização espacial onde objetos surgem, intensidade de sons, de brilho e das imagens, além de diversas características de frequência, como timbre e cor. Quanto maior for o número de parâmetros que podem ser modificados, maior será a interatividade de um determinado sistema.

Mapeamento: refere-se à capacidade de um sistema em mapear os controles para possíveis mudanças em determinado ambiente de forma natural e previsível. Essa capacidade de mapeamento pode ser completamente arbitrária e sem relação com a função desempenhada. Por exemplo, ao balançar um dedo da mão, pode-se aumentar o volume do som da televisão. Ou o mapeamento pode ser completamente natural, como ao dirigir um volante em um videogame, pode fazer o "carro virtual" na tela mover-se conforme os movimentos.

Sims (1997) explica e apresenta níveis de interatividade por ele identificados, com a suposição de que, quanto maior o nível, melhor o produto. Nesse sentido, apresenta níveis de interatividade por ele compilados, que podem ser utilizados como base para a análise de sistemas interativos.

1- Interatividade do Objeto: refere-se a uma aplicação em que os objetos como botões, etc. -, são ativados através do uso de um mouse ou outro dispositivo que acione o sistema, de forma que quando um usuário "clica" sobre o objeto, o sistema oferece resposta através de áudio visual.

2- Interatividade Linear: refere-se a aplicações em que o usuário é capaz de mover para frente ou para trás, através de uma sequência linear predeterminada; essa classe de interação não oferece feedback específico de resposta às ações do usuário.

3- Interatividade de Suporte: refere-se à facilidade em que o usuário recebe suporte ao desempenho realizado, que pode estar relacionado às mensagens de ajuda.

4- Interatividade de Atualização: refere-se aos componentes de aplicação individual ou eventos em que um diálogo é iniciado entre o usuário, e o conteúdo, gerado por computador ou sistema.

5 - Interatividade de Construção: refere-se a um ambiente em que o usuário é obrigado a manipular objetos componentes para atingir seus objetivos específicos.

6- Interatividade Reflexiva: refere-se à classe de interação (elaboração proativa), para atender às muitas situações em que os designers desejam incluir respostas de texto para instruções ou perguntas.

7- Simulação de Interatividade: refere-se ao papel do usuário como controlador ou de operador, onde seleções individuais determinam a sequência de treinamento.

8- Interatividade de Hiperligação: refere-se ao acesso e navegação do usuário no sistema com riqueza de informações, e pode "navegar" à vontade com base nesse conhecimento.

9- Interatividade Contextual Não-Imersiva: esse conceito condiz e estende-se aos vários níveis de interatividade: refere-se à capacidade do usuário de navegar através uma série de conteúdos que orientam a navegação em um ambiente virtual.

10- Interatividade Imersiva Virtual: a interatividade virtual imersiva fornece um ambiente interativo em que o usuário está projetado em um mundo gerado por computador, que responde ao movimento e ações individuais. 
Para Tori (2010) existe uma quantidade muito grande de classificações e definições para os termos interatividade e interação. Nesse sentido, o autor explica a diferença da definição de cada termo: a interação é exercida entre dois elementos, de forma que haja interferência mútua no comportamento de ambos inter atores. Já a "a interatividade é a percepção da capacidade, ou potencial, de interação propiciada por determinado sistema ou atividade." (TORI, 2010, p. 5).

Através dos elementos descritos a seguir, pode-se aumentar a percepção de interatividade de determinado sistema:

Frequência: periodicidade da ocorrência de oportunidades de interação; uma atividade que permite interrupção a qualquer instante - frequência contínua - certamente será percebida como possuindo mais interatividade do que se a interação fosse limitada a apenas determinados momentos.

Abrangência: conjunto de opções disponíveis ao interator nos momentos de interação; a abrangência pode ser representada por um menu de opções discretas ou de forma implícita, como as possibilidades de movimentação e atuação de um avatar num mundo virtual.

Significado: componente subjetivo da interatividade; quanto mais importante e significativa for determinada ação para o participante de uma atividade, ou usuário de um sistema, menor será sua percepção de baixa frequência ou de pouca abrangência; um fã que aguarda o show inteiro pela possibilidade de ser sorteado e poder subir ao palco para abraçar seu ídolo certamente terá mais sensação de interatividade que o aluno aguardando a hora do sinal para sair de uma aula da qual não esteja efetivamente presente (LAUREL, 1989 apud TORI, 2010, p. 5).

Tori (2010) considera como "tecnologias interativas" todas as ferramentas e recursos tecnológicos que estão diretamente relacionados aos sistemas e ambientes providos do meio informatizado. Ao analisar e observar a discussão dos autores, suas abordagens se complementam, de forma que ambos contribuem para uma constante evolução da definição do conceito e aplicação da boa interatividade de sistemas.

Rogers, Sharp e Preece (2013) conceituam o design através de tipos de interação que auxiliam em mudanças, ou ainda em requisitos para a boa experiência do usuário.

Instrução: Trata-se do estilo em que os usuários emitem instruções a um sistema, isso pode ser feito de diversas maneiras, como: digitar comandos, selecionar opções de menus em um ambiente de janelas ou em uma tela multitoque, pressionar botões ou usar combinações de teclas de função.

Conversação: É o tipo de interação em que os usuários podem gerar um diálogo com um sistema, isso pode ocorrer de forma em que os usuários podem falar por meio de uma interface ou enviar perguntas para as quais o sistema responde via texto ou saída de voz.

Manipulação: Trata-se das formas de manipulação, ou seja, a forma em que usuários interagem com os objetos em um espaço virtual ou físico (p. ex., abrir, segurar, fechar).

Exploração: É o estilo em que os usuários se movem por um ambiente virtual, que incluem mundos 3D e sistemas de realidade aumentada e de realidade virtual e espaços físicos que utilizam tecnologias baseadas em sensores e salas ou ambientes inteligentes.

Os tipos de interação citados por Rogers, Sharp e Preece (2013) referem-se às maneiras pelas quais uma pessoa pode interagir com um sistema, produto ou com a sua aplicação. Cada um dos tipos de interação permite que designers analisem interfaces ou formulem modelos conceituais de interfaces. 
Diante do exposto pelos autores aqui abordados, na seção seguinte é apresentada a metodologia de pesquisa do presente estudo.

\section{METODOLOGIA}

A metodologia utilizada para o estudo é de cunho qualitativo - empírico descritivo, baseando-se em análise bibliográfica (CRESWELL, 2010; MAXWELL, 2005). Com base no referencial teórico, foi adotada a técnica de análise de conteúdo, que resultou no quadro referencial. Esse quadro foi posteriormente validado por especialistas mediante a técnica de análise de inspeção - que tem como objetivo a validação de conteúdo e a validação de aparência, conforme descrito na presente seção.

A primeira etapa do estudo é baseada no referencial teórico, que sustentou os dados para a concepção das etapas seguintes da pesquisa.

A segunda etapa da pesquisa é concentrada no atendimento do objetivo do presente estudo, que se trata do quadro referencial para a análise de interatividade de sites de e-commerce do ramo da moda (vestuário, especificamente) em smartphone. 0 desenvolvimento do mesmo se constituiu de etapas de seleção e agrupamento de dados e conceitos teóricos, pesquisados ao longo da primeira etapa do estudo. Essa etapa foi constituída mediante o método de análise de conteúdo.

A última etapa se refere à validação do quadro referencial por quatro especialistas, sendo três Pesquisadores: um Doutor em Comunicação, um Mestre em Design e um Mestre em Moda, todos atuantes na área do ensino. A escolha pelos especialistas deu-se pela formação dos mesmos e especialidade na área de pesquisa, identificadas previamente através do Currículo disponível na plataforma Lattes aderentes ao presente estudo. O quarto avaliador é um profissional especialista e atuante no mercado, como desenvolvedor de e-commerce, formado em Design, atua há sete anos no desenvolvimento de e-commerce de diferentes segmentos - dentre eles, o segmento da moda (vestuário).

A validação deu-se através da análise de um caso; baseando-se no quadro referencial desenvolvido, os especialistas realizaram a inspeção em dois sites de $e$ commerce e validaram as questões expostas no quadro e em sua estrutura - ou seja, a validade de conteúdo e a validade de aparência.

Para isso, foram escolhidos dois sites de e-commerce. Cabe destacar que, na presente pesquisa, as duas empresas tratadas foram identificadas como $E$-commerce $A$ e E-commerce $\mathrm{B}$, a fim de preservar o anonimato das organizações no estudo. $\mathrm{O}$ critério de seleção para a definição dos sites foi o da conveniência, sendo ambos escolhidos com base na estrutura dos referidos espaços, e ainda, pelo conhecimento de que se tratam de grandes lojas do ramo do vestuário que operam com e-commerce. A escolha dessa técnica se justifica pela possibilidade de uma avaliação real - ou seja, o especialista, diante das questões, identifica especificidades não previstas e divergências a serem corrigidas.

Com a avaliação realizada pelos especialistas, foram realizadas adequações quanto ao quadro, sua estrutura e conteúdo e, por fim, é apresentado o resultado final. 


\section{ELABORAÇÃO DO QUADRO REFERENCIAL PARA ANÁLISE DA INTERATIVIDADE}

Considerando-se que o quadro referencial foi construído partir da literatura existente, utilizou-se do método de análise de conteúdo, que tem como objetivo descrever e interpretar o conteúdo abordado no referencial teórico da pesquisa. Essa análise ajuda a reinterpretar o conteúdo e a atingir a compreensão de seus significados (MORAES, 1999).

Bardin (2006) explica que a análise de conteúdo possibilita a elaboração de categorias descritivas, de forma que a compreensão do sentido do discurso e a escolha do contexto da mensagem tornam-se requisitos fundamentais para a investigação.

Portanto, a fim de atender objetivo geral do estudo em propor um quadro referencial para análise de sites de e-commerce do ramo da moda via smartphone que prevê fatores e critérios que devem ser considerados em nível de interatividade -, seguiu-se o seguinte processo para a análise do conteúdo:

Pré-análise das informações: caracteriza-se pelos temas e documentos escolhidos para o estudo. A etapa de pré-análise do presente estudo foi baseada no referencial teórico - que buscou identificar, através da literatura, informações referentes aos fatores e critérios relevantes para a análise de interatividade.

Descrição analítica: com base na pré-análise, nessa etapa consideraram-se as especificidades de e-commerce, as etapas de navegação e os níveis de interatividade; essas unidades foram separadas, a fim de realizar a etapa seguinte, que se trata da categorização do conteúdo.

Categorização do conteúdo: A caracterização de conteúdo, conforme Bardin (2006) é uma operação de classificação, na qual os elementos (conteúdo, discurso, temas) são diferenciados e reagrupados. Nesse sentido, a presente pesquisa buscou selecionar e agrupar cada uma das etapas que viram a compor o quadro referencial, na etapa de categorização - a principal etapa do estudo. A categorização é um procedimento de agrupar dados considerando a parte comum existente entre eles. Há diferentes classificações para realizar a categorização do conteúdo. Na presente pesquisa, considerou-se o sentido dos enunciados de cada item analisado (MORAES, 1999). O processo de categorização deve ser entendido como um processo para a redução de dados, que representa o resultado de uma síntese, destacando, neste processo, os seus aspectos mais importantes (MORAES, 1999).

Interpretação referencial e tratamento dos resultados: por fim, a etapa de interpretação e tratamento dos resultados foi realizada através do método de inspeção, que teve como objetivo a validação do instrumento de pesquisa, conforme sugerido por Bardin (2006).

A validade ou pertinência dos resultados obtidos exige que os dados contidos no instrumento de pesquisa sejam significativos e úteis em termos do trabalho proposto, levando em consideração a problemática, objetivos e fundamentação teórica (MORAES, 1999).

\subsection{Resultado: Quadro referencial de análise}

A avaliação do quadro referencial por especialistas deu-se através do método de inspeção, de forma que, diante do quadro referencial desenvolvido, os especialistas realizaram a inspeção em site de e-commerce, validando as questões expostas no quadro e na sua estrutura, conforme se descreve a seguir:

Procedeu-se à a análise da interatividade de dois sites de e-commerce, denominados na pesquisa de E-commerce A e E-commerce B. O método de inspeção 
tem como objetivo identificar problemas de usabilidade ou de interatividade no design de uma interface de usuário e, diante desses problemas, gerar sugestões de adaptação - além de propor recomendações no sentido de eliminar os problemas, melhorando assim a interface, o que pode ser realizado em qualquer fase de um sistema implementado ou não (ROCHA; BARANAUSKAS 2003).

Nesse sentido, Rogers, Sharp e Preece (2013) explicam que tal tipo de avaliação não envolve o usuário, pois os métodos de inspeção buscam prever o comportamento do usuário e, dessa forma, identificar possíveis problemas. Além disso, a sua realização ocorre em ambientes em que o pesquisador tende a navegar ou a modelar a forma pela qual a interface provavelmente será utilizada pelo usuário.

Para a técnica de inspeção, os avaliadores podem ser especialistas, consultores de desenvolvimento de software, especialistas em determinado padrão de interface, usuários finais, etc. (ROCHA; BARANAUSKAS 2003).

Nesse sentido, o quadro referencial foi enviado a três especialistas professores/pesquisadores atuantes na área do ensino, sendo um Doutor em Comunicação, um Mestre em Design e um Mestre em Moda e um profissional desenvolvedor de e-commerce. A participação dos quatro especialistas objetivou a validação dos requisitos, conceitos teóricos e estrutura, bem como, da avaliação de um profissional atuante na área, atendendo questões restritas a aspectos técnicos.

Com relação ao número de especialistas escolhidos para o estudo, considerouse a abordagem proposta por Rogers, Sharp e Preece (2013), em que os autores sugerem que neste tipo de pesquisa sejam envolvidos de três a cinco avaliadores, a fim de ter maior número de resultados.

A validade de um instrumento de pesquisa está relacionada com a precisão do instrumento no quesito de medir o que se propõe medir. Portanto, um instrumento é considerado válido quando sua construção e aplicabilidade mensura a variável daquilo que realmente se pretende mensurar (SAMPIERI, COLLADO e LUCIO, 2013).

As técnicas utilizadas para a avaliação do presente estudo constituem-se na validade de conteúdo e na validade de aparência. A validade de conteúdo refere-se ao grau em que um instrumento determina a representatividade de itens que expressam um conteúdo, baseada no julgamento de especialistas na área específica. Portanto, a validação de conteúdo determina se o conteúdo expresso em um instrumento de medida explora, de fato, os quesitos para mensuração do fenômeno a ser investigado; já a validação de aparência proporciona a análise sobre a relevância e adequação dos itens expostos no instrumento (JUNIORI e MATSUDA, 2012; SAMPIERI, COLLADO e LUCIO, 2013).

Nesse sentido, o quadro referencial foi enviado aos especialistas através de email. As instruções indicavam que os especialistas deveriam primeiramente acessar aos sites de e-commerce via smartphone através do navegador, pois as lojas selecionadas não possuem aplicativo específico; em seguida, navegar pela interface dos sites de e-commerce via smartphone e, de acordo com as questões expostas no quadro referencial, realizar a inspeção do site. Foi solicitado que a inspeção nos sites de e-commerce via smartphone fosse realizada no mínimo duas vezes, de forma que se identificasse o maior número possível de dados na navegação como subsídios para identificação de itens a serem observados na avaliação do quadro. 
Quadro 1: Resultado do estudo: quadro referencial para análise de interatividade de sites de $e$ commerce do ramo da moda (vestuário) via smartphone.

\begin{tabular}{|c|c|c|c|c|c|}
\hline \multicolumn{6}{|c|}{ I"ETAPA - ANALISE ETAPAS DE NAVEGACAO } \\
\hline ANÁLISE ETAPAS DE NAVEGAÇÄO & $\begin{array}{l}\text { Muito abaxo } \\
\text { das } \\
\text { expectativas }\end{array}$ & $\begin{array}{l}\text { Abaico das } \\
\text { expectativas }\end{array}$ & Neutro & $\begin{array}{l}\text { Conforme } \\
\text { esperado }\end{array}$ & $\begin{array}{l}\text { Mais do que } \\
\text { esperado }\end{array}$ \\
\hline $\begin{array}{l}\text { 1-Acessaro catalogo de produtos: A escoling de um } \\
\text { produto é realizada com agilidade através dos filtros } \\
\text { disponiveis porcategorias? (Ex: de marca, de cores, de } \\
\text { tamanho, de estampa e de estilo) }\end{array}$ & 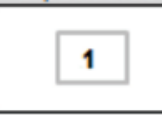 & 2 & 3 & 4 & 5 \\
\hline $\begin{array}{l}\text { 2. Buscar por produtos: O e-commerce oferece } \\
\text { campo para a busca por produtos com agilidade? }\end{array}$ & 1 & 2 & 3 & 4 & 5 \\
\hline $\begin{array}{l}\text { 3- Selecionar um produto: Ao selecionsr um produto o } \\
\text { e-commerce oferece links com opçöes de diferentes } \\
\text { cores, tamanhos e estampas com facilidade de escohs? }\end{array}$ & 1 & 2 & 3 & 4 & 5 \\
\hline $\begin{array}{l}\text { 4-Visualizaçá de detalhes dos produtos: Oe- } \\
\text { commerce oferece detalhes dos produtos através de } \\
\text { imagens de alts qualidade? } \\
4.1 \text { - O e-commerce oferece orientação e descriçäo }\end{array}$ & 1 & 2 & 3 & 4 & 5 \\
\hline 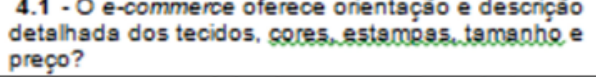 & 1 & 2 & 3 & 4 & 5 \\
\hline $\begin{array}{l}\text { 5-Acessar o carninho decompras: E possivelexdur } \\
\text { ou incluir produtos de forma ágil do carrinho de } \\
\text { compras? }\end{array}$ & 1 & 2 & 3 & 4 & 5 \\
\hline $\begin{array}{l}6 \text { - Identificaçáo do Usuário: O processo de } \\
\text { identificaçäo (através do legia ou cadastro) foi rápido e } \\
\text { ágil? }\end{array}$ & 1 & 2 & 3 & 4 & 5 \\
\hline $\begin{array}{l}\text { 6.1-O e-commerce oferece informações claras, sobre } \\
\text { as formas de pagamento e de entrega? }\end{array}$ & 1 & 2 & 3 & 4 & 5 \\
\hline $\begin{array}{l}7 \text { - Finalizaçáo: Do cadastro ate as opçoes de } \\
\text { pagamento o sistema foi eficiente? }\end{array}$ & 1 & 2 & 3 & 4 & 5 \\
\hline $\begin{array}{l}\text { 8-Confirmaçác: Oe-commerce oferece uma tela de } \\
\text { confirmação da compra, com revisäo de pedido e } \\
\text { formas de pagamento? }\end{array}$ & 1 & 2 & 3 & 4 & 5 \\
\hline \multicolumn{6}{|c|}{ 2 ETAPA - ANALISE DOS NIVEIS DE INTERATIVIDADE } \\
\hline ANALISE DO NIVEL DE INTERATIVIDADE & $\begin{array}{l}\text { Nivel muito } \\
\text { baixo }\end{array}$ & Nivel baico & Nivel Regular & Nivel alto & \begin{tabular}{|c|}
$\begin{array}{c}\text { Nivel muito } \\
\text { alto }\end{array}$ \\
\end{tabular} \\
\hline $\begin{array}{l}\text { 1. O sistema possibilita de forma naturale rapida a } \\
\text { manipulação de links e filtros (ex; abrir uma imagem, } \\
\text { selecionar e clicar sobre um filtro)? }\end{array}$ & 1 & 2 & 3 & 4 & 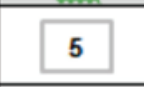 \\
\hline $\begin{array}{l}2 \text { - O sistema oferece feedback das atividades } \\
\text { realizadas, seja com áudio, video ou mensagens de } \\
\text { ajuds? }\end{array}$ & 1 & 2 & 3 & 4 & 5 \\
\hline $\begin{array}{l}\text { 3- O sistema possibilitou a naveg } 9 c ̧ \Im 0 \text { sem interrupçöes } \\
\text { e com bos frequếncia? }\end{array}$ & 1 & 2 & 3 & 4 & 5 \\
\hline $\begin{array}{l}\text { 4-O sistema foiclaro e transparente nas informaçōes } \\
\text { prestadas, oferecendo segurança durante a navegaçäo } \\
\text { no site? }\end{array}$ & 1 & 2 & 3 & 4 & 5 \\
\hline $\begin{array}{l}\text { 5- O sistema oferece controle e autonomis de forma } \\
\text { eficaz, possibilitando as açöes de interatividade? }\end{array}$ & 1 & 2 & 3 & 4 & 5 \\
\hline $\begin{array}{l}\text { 6- O sistema oferece uma interface com design e } \\
\text { conteúdos bem elaborados, que orientama navegoçäo? }\end{array}$ & 1 & 2 & 3 & 4 & 5 \\
\hline $\begin{array}{l}\text { 7- O sistema oferece opçoes de facil acesso para } \\
\text { gutostendimento? }\end{array}$ & 1 & 2 & 3 & 4 & 5 \\
\hline $\begin{array}{l}\text { 8-0 sistema fornece uma navegação linear, ou seja, a } \\
\text { navegação possibilitou um único caminho? }\end{array}$ & 1 & 2 & 3 & 4 & 5 \\
\hline $\begin{array}{l}\text { 9-O sistema funciona em duasdireçoes, o usuaro pode } \\
\text { ir e voltar em sua navegaçäo sem maiores dificuldades } \\
\text { na interface de smartphone? }\end{array}$ & 1 & 2 & 3 & 4 & 5 \\
\hline $\begin{array}{l}\text { 10-O sistema oferece velocidade na interaçäo e rapido } \\
\text { tempo de resposta em relação às açōes realizadas } \\
\text { durante a navegaçäo? }\end{array}$ & 1 & 2 & 3 & 4 & 5 \\
\hline $\begin{array}{l}\text { 11-O site de e-commerce mostrou-se com design } \\
\text { adaptado e funcional para a bos interatividade na } \\
\text { interface de smartphone? }\end{array}$ & 1 & 2 & 3 & 4 & 5 \\
\hline
\end{tabular}

Portanto, diante da adoção da análise de conteúdo e da validação por especialistas apresenta-se a seguir o resultado do estudo, o quadro referencial para análise da interatividade de sites de e-commerce do ramo da moda em smartphone.

O quadro referencial é aqui apresentado no formato de um conjunto de questões, que visam identificar o nível de interatividade de um site mediante as classificações baseadas na escala de Likert. 


\section{CONCLUSÃO}

Com objetivo principal de propor um quadro referencial para análise da interatividade de sites de e-commerce do ramo da moda-vestuário via smartphone, o presente trabalho teve como problema investigar quais fatores e critérios que deveriam ser considerados em nível de interatividade para a análise de sites de $e$ commerce do ramo da moda-vestuário.

Com relação ao estudo quanto aos níveis de interatividade existentes na literatura, observou-se que nem todos eram adequados ao quadro referencial, no contexto de mobile commerce. Com isso, considerando os métodos de seleção, agrupamento e análise de conteúdo, foi possível agrupar os níveis que se enquadravam com o objetivo e selecionar os que estavam adequados para responder ao problema de pesquisa.

Deste modo, a principal contribuição do estudo concentra-se no resultado do quadro referencial para análise da interatividade de sites de e-commerce do ramo da moda via smartphone, oferecendo aos pesquisadores da área, desenvolvedores e designers uma ferramenta que possibilite, de modo mais estruturado, levantamento e identificação de requisitos de interatividade para sites de e-commerce de Moda.

O trabalho contribui ainda com a identificação de fatores e critérios observados ao longo do estudo, que devem ser considerados em relação a boa interatividade em sites de e-commerce do ramo da moda.

Sugere-se, como trabalhos futuros, a aplicação do quadro referencial estabelecido no estudo em uma coleta de dados mais abrangente para medir a complexidade de alcance de interatividade de sites de e-commerce do ramo da moda via smartphone, a fim de gerar dados quanto ao nível de interatividade existente nestes meios.

Outro componente que pode ser analisado em pesquisas futuras é a adaptação do quadro referencial para a análise do usuário final, quanto aos sites de e-commerce. Desta forma, poder-se-ia investigar o nível de interatividade sob o ponto de vista do usuário, estabelecendo uma coleta com relevância em relação à quantidade de dados (sites) analisados.

\section{REFERÊNCIAS}

BARDIN, L. Análise de conteúdo. Lisboa: Edições 70, 2006.

BORTOLÁS, Natália Ordobás; GONÇALVES, Berenice, VIEIRA, Milton Horn. Análise da interatividade em sites de e-commerce - Interactivity analysis on e-commerce websites. Projética Revista Científica de Design, Londrina, V.3, N.2, 2012.

CRESWELL, John W. Projeto de Pesquisa: métodos qualitativo, quantitativo e misto. 3a ed. Porto Alegre: Artmed, 2010.

JUNIOR, Jose Aparecido Bellucci; MATSUDA, Laura Misue. Construção e validação de instrumento para avaliação do Acolhimento com Classificação de Risco. Rev Bras Enferm, Brasília 2012 set-out; 65(5): 751-7.

MORAES, Roque. Análise de conteúdo. Revista Educação, Porto Alegre, v. 22, n. 37, p. 7-32, 1999.

RAFAELI, Sheizaf. Interactivity: From New Media to Communication,1988. 
RAINER, JR, R., Kelly; CEGIELSKI, Casey, G. Introdução a sistemas de informação. Apoiando e transformando negócios na era da mobilidade. 3a.ed. - Rio de Janeiro: Elsevier, 2011.

ROCHA, Heloísa Vieira da; BARANAUSKAS, Maria Cecília Calani. Design e Avaliação de Interfaces Humano-Computador. Instituto de Computação. Universidade Estadual de Campinas, Campinas, SP: NIED/UNICAMP, 2003.

ROGERS, Yvonne; SHARP, Helen; PREECE, Jennifer. Design de interação: além da interação humano - computador. 3. Ed. - Porto Alegre: Bookman, 2013.

SAMPIERI, Roberto Hernandez; COLLADO,Carlos Fernández; LUCIO, María del Pilar Baptista. Metodologia de Pesquisa. 5a ed. - Porto Alegre: Penso, 2013.

SIMS, Roderick. Interactivity: a Forgotten Art?. Faculty of Education. University of Technology, Sydney. 1997. Disponível em: <

http://www2.gsu.edu/ wwwitr/docs/interact/>. Acesso em: 20 de set. 2014.

STEUER, Jonathan. Defining Virtual Reality: Dimensions determining telepresence. Department of Communication, Stanford University. This paper originally appearred in the Journal of Communication, 4(24) (Autumn, 1992), 73-93, 1993

STAIR, Ralph. M; REYNOLDS, George W. Princípios de sistemas de informação. 9.ed. São Paulo: Cengage Learning, 2012.

TORI, Romero. A presença das tecnologias interativas na educação. Revista de Computação e Tecnologia da PUC-SP - Departamento de Computação/FCET/PUC-SP, Vol. II, No: 1, 2010. 